\title{
Gummatous penile ulceration and generalised lymphadenopathy in homosexual man: case report
}

\author{
V S KITCHEN, * T COOK, $†$ A DOBLE, $\ddagger$ J R W HARRIS* \\ From the Departments of *Genitourinary Medicine, $\uparrow$ Histopathology, and $\ddagger$ Urology, St Mary’s Hospital, \\ London
}

SUMMARY A case of granulomatous penile ulceration associated with generalised lymphadenopathy in a homosexual man aged 43 is presented. His serum gave positive results to tests for syphilis, but was negative for antibody to human immunodeficiency virus (HIV). His condition responded briskly to treatment with procaine penicillin, and we conclude that the clinical features were attributable to infection with Treponema pallidum.

Gummatous ulceration of the penis is now exceptionally rare. Catteral et al, reporting five cases in 1957, noted that only 11 other cases had been reported during the previous 40 years. ${ }^{2}$ This condition was, however, well recognised, in the era before treatment with arsenic, by doctors who adopted the term pseudochancre redux for lesions that often resembled the chancre of initial infection.

In the case we report, the clinical picture was complicated by the presence of pronounced generalised lymphadenopathy, which is nowadays often mistakenly considered to be synonymous with human immunodeficiency virus (HIV) infection in members of the population who are at risk.

\section{Case report}

A German homosexual man aged 43 presented to the Praed Street Clinic on 29 July 1987, having been referred by Northwick Park Hospital's urology department with a 16 month history of painless penile ulceration and recent positive results to syphilis serological tests. Eighteen months previously, in February 1986, he had developed a small painless left scrotal ulcer, which had resolved spontaneously during the subsequent two months. As that lesion healed, a similar lesion appeared at the tip of the glans penis. The second lesion initially resembled a papule and subsequently enlarged and ulcerated. The severity of the ulcer fluctuated slightly with time, and when the

Address for reprints: Dr V S Kitchen, Praed Street Clinic, St Mary's Hospital, London W2 INY

Accepted for publication 23 December 1987 lesion was at its worst the patient noticed a thick white urethral discharge.

He gave no history of syphilis, and denied any previous episodes of penile ulceration or skin rashes. He had lived in the United Kingdom for eight years, but before that had stayed in Bangkok for several months, during which time he had had several homosexual contacts. He had been monogamous while living in the United Kingdom, and had had no sexual contact since the development of his penile lesion.

\section{EXAMINATION}

An atrophic scar $1 \mathrm{~cm}$ in diameter was visible on the left scrotum. The urethral meatus was surrounded by a well demarcated, solitary, painless ulcer, measuring $2 \mathrm{~cm}$ in diameter, with areas of basal sloughing (fig 1). Enlarged, non-tender lymph nodes measuring $1-2 \mathrm{~cm}$ in diameter were palpable in the right cervical, left axillary, and left inguinal regions. No other abnormality was seen.

\section{INVESTIGATIONS}

Dark field examination of the ulcer showed no Treponema pallidum on four consecutive days. Microscopy of a direct smear from the base of the ulcer gave negative results for Calymmatobacter granulomatis, Mycobacterium tuberculosis, and Entamoeba histolytica. Specimens taken from the ulcer were cultured for $C$ granulomatis, $M$ tuberculosis, Chlamydia trachomatis, and Haemophilus ducreyi, and no evidence of these organisms was found. Culture did yield a moderate growth of Staphylococcus aureus and a group B streptococcus, Lancefield type G.

Urethral culture for Neisseria gonorrhoeae and 


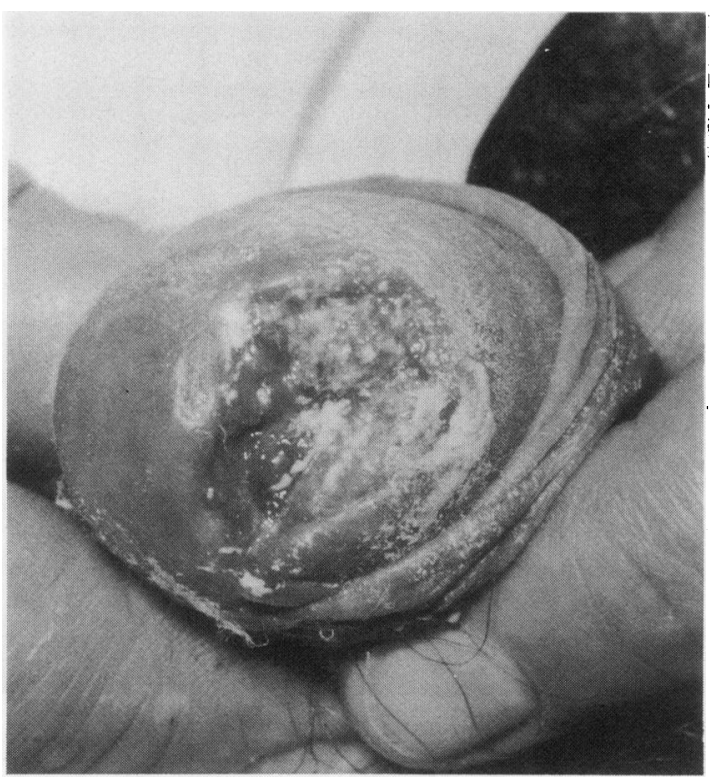

Fig 1 Appearance of penile ulcer before treatment.

culture of four early morning urine specimens for $M$ tuberculosis gave negative results.

A full blood count showed a haemoglobin concentration of $171 \mathrm{~g} / \mathrm{l}$ and a white cell count of $7.2 \times 10^{9} / \mathrm{l}$, with a normal differential. Radiological examination of the chest and long bones showed no abnormality.

A Mantoux test using $10 \mathrm{IU}$ human tuberculin purified protein derivative (PPD) and a delayed cutaneous hypersensitivity skin test, bearing the standard panel of antigens, were planted simultaneously. The results were read at 48 hours, when the Mantoux response was positive at $20 \mathrm{~mm}$, and the cumulative delayed cutaneous hypersensitivity skin test score totalled $41 \mathrm{~mm}$, with a $10 \mathrm{~mm}$ response to human tuberculin, a $15 \mathrm{~mm}$ response to diphtheria toxin, and a negative response to glycerine (fig 2).

Positive results were found by the following serological tests for syphilis: the fluorescent treponemal antibody absorption (FTA-ABS) test, the $T$ pallidum haemagglutination assay (TPHA), and the Venereal Disease Research Laboratory (VDRL) test at a titre of $1 / 1024$. The test for antibody to HIV, using an enzyme linked immunosorbent assay (ELISA) kit (Abbott), gave a negative result. An immunofluorescent antibody test for $E$ histolytica (performed in the department of protozoology at the London School of Tropical Medicine) gave a negative result.

Examination of the cerebrospinal fluid (CSF) gave positive results to the TPHA and FTA-ABS test and a negative result to the VDRL test. The fluid was acellular, with a normal protein content.
Biopsy of the penile lesion showed an area of ulceration, the surface of which was covered with necrotic slough (fig 3). Microscopy of tissue sections taken through the base of the ulcer showed multiple epithelioid granulomata with prominent giant cells (fig 4). Areas of eosinophilic necrosis were present in the granulomata. The connective tissue surrounding the granulomata contained a proliferation of capillary vessels and a diffuse lymphocytic infiltrate. Plasma cells were not a prominent feature.

No micro-organisms were seen on Gram, periodic acid-Schiff, Ziehl-Nielsen, or Warthin-Starry stains.

\section{TREATMENT}

On 19 August 1987 we started treatment with daily intramuscular injections of procaine penicillin 600000 IU for two weeks. The patient developed a widespread erythematous macular rash suggestive of allergy to penicillin towards the end of that course, and treatment was changed to oral doxycycline $300 \mathrm{mg}$ a day for a further week. On review at two weeks, the penile

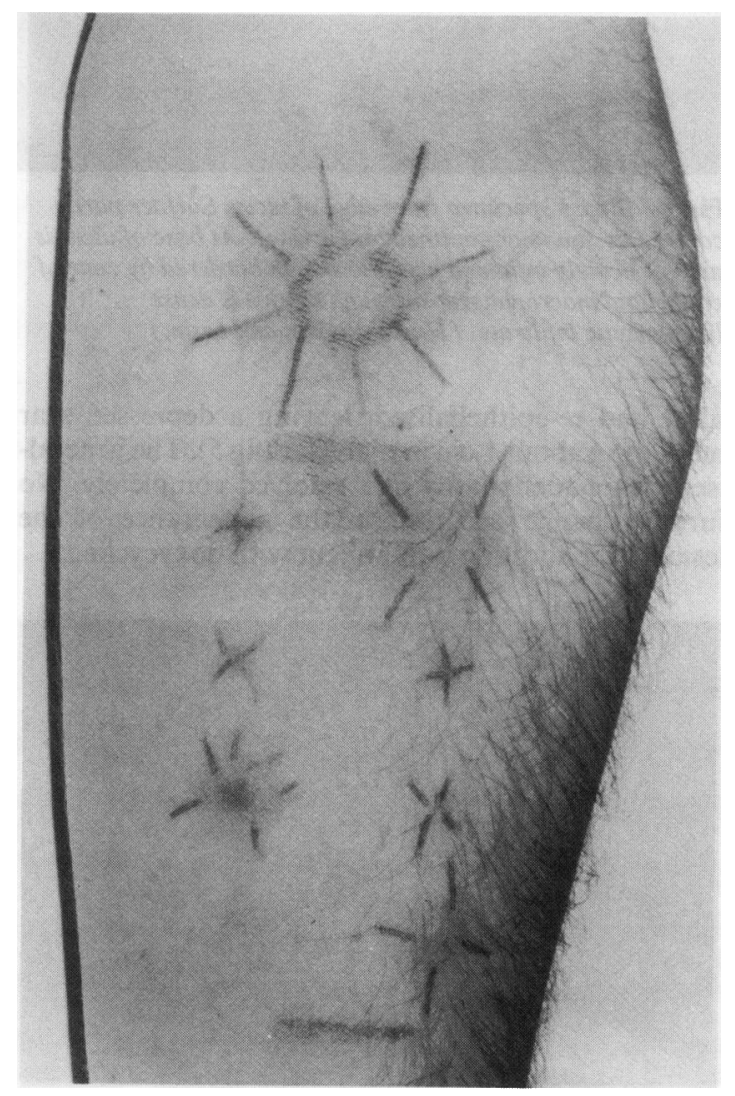

Fig 2 Mantoux test (above) and delayed cutaneous hypersensitivity skin test responses at 48 hours. 


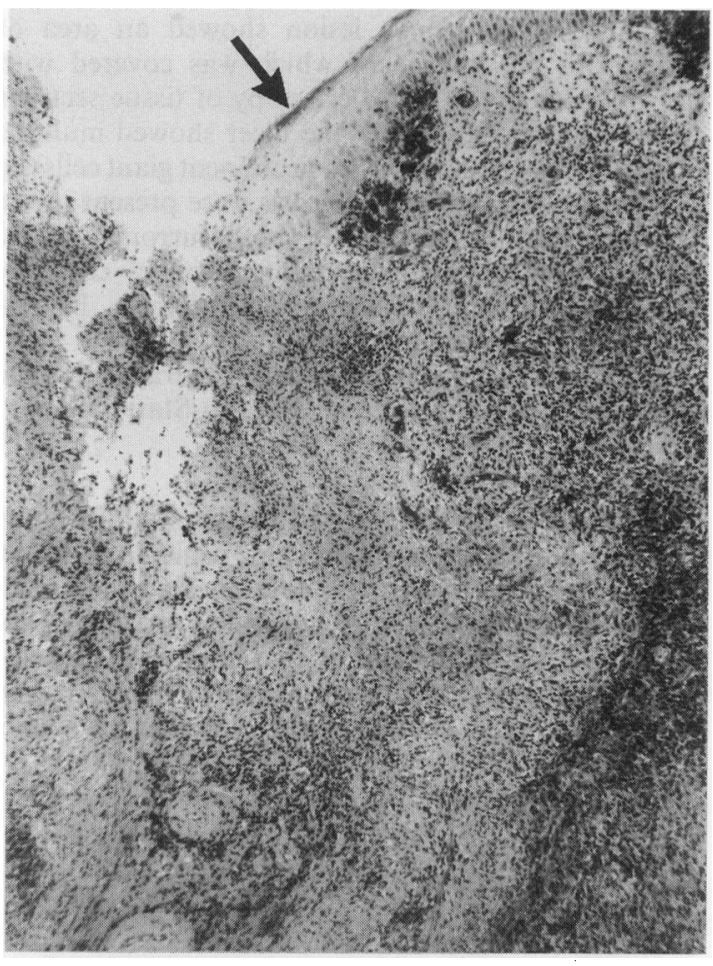

Fig 3 Biopsy specimen from edge of ulcer. Surface partly covered by squamous epithelium (arrow). At base of ulcer is area of acutely inflamed necrotic slough bordered by zone of epithelioid macrophages; surrounding this is dense lymphocytic infiltrate. (Haematoxylin and eosin.)

ulcer had re-epithelialised, leaving a depressed scar measuring about $1 \mathrm{~cm}$ in diameter (fig 5). The generalised lymphadenopathy had resolved completely. No further change occurred in the appearance of the lesion after additional treatment with doxycycline.

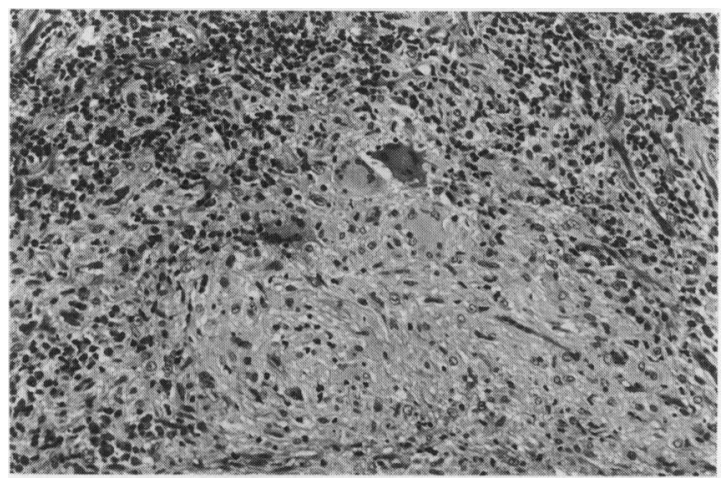

Fig 4 Epithelioid granuloma containing multinucleate giant cells. (Haematoxylin and eosin.)

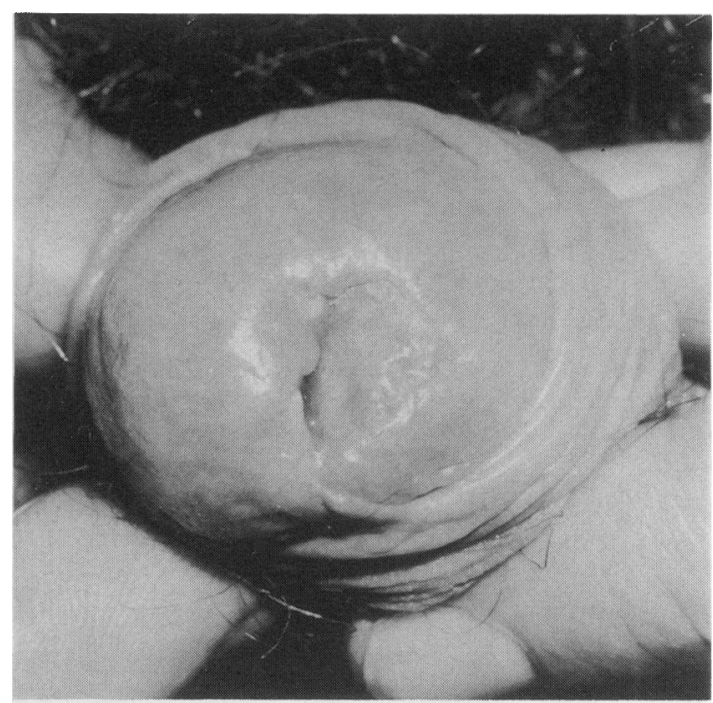

Fig 5 Appearance of penile lesion after treatment.

\section{Discussion}

This patient presented with a chronic painless penile ulcer that had many of the chronological, macroscopic, and microscopic features of a gumma. In association with this lesion, however, he had features strongly suggestive of secondary syphilis, namely generalised lymphadenopathy and a positive VDRL test result at a titre $1 / 1024$. Complete resolution of the lymphadenopathy after standard antitreponemal treatment implied that this lymphadenopathy was an integral feature of the underlying condition, and unlikely to be due to another disease.

One explanation for the coexistence of features of secondary and tertiary syphilis in our patient may be that he had secondary syphilis with a late monorecidive lesion that had many characteristics of a gumma, including that of rapid healing with tissue loss in response to treatment. ${ }^{3}$ Such lesions have been documented as occurring up to four years after initial infection. In 1946 Stokes et al recognised that these lesions were more likely to resemble gummata the longer after initial infection that they occurred. ${ }^{3}$

In his follow up of the Boeck-Bruusgaard cohort, Gjestland reported that $23.6 \%$ of untreated patients suffered at least one secondary relapse, and he concluded that monorecidive lesions were a normal feature of untreated syphilis. ${ }^{45}$.

An alternative explanation is that our patient initially became infected many years before presentation, and may then have been reinfected by his recent longstanding sexual partner, which resulted in the 
development of a gummatous lesion at the site of reinfection.

It is generally accepted that the gumma represents a maximum delayed hypersensitivity response to a few treponemal organisms. These treponemes may be endogenous, in untreated or inadequately treated patients, or exogenous, in patients with prior immune sensitisation who become reinfected. ${ }^{6}$ In 1956 Magnuson et al studied the effects of treponemal innoculation into volunteers in Sing Sing prison, and found that gummatous skin lesions developed at the site of inocculation in 10 out of 26 people with a history of syphilis. ${ }^{7}$ The patient we describe showed a florid delayed hypersensitivity skin response to multiple standard antigens, and clearly possessed the ability to mount a brisk $T$ cell response when challenged. In this case, the possibility of reinfection as the cause of the clinical features observed is not clear, as details of the patient's most recent sexual partner were not available.

We thank Mr M Burke, Consultant Surgeon, Department of
Urology, Northwick Park Hospital, Harrow, Middlesex, for refering the patient, and Miss Catrina Donegan for kindly typing the manuscript.

\section{References}

1 Catterall RD, Collins CD, Redmond A. Gumma of the penis. British Journal of Venereal Diseases 1957;33:160-4.

2 Al-Egaily S. Gumma of the testis and penis. British Journal of Venereal Diseases 1977;53:257-9.

3 Stokes JH, Beerman H, Ingraham NR. Modern clinical syphilology. 3rd ed. Philadelphia: Saunders, 1946:638.

4 Gjestland T. The Oslo study of untreated syphilis: an epidemiologic investigation of the natural cause of syphilitic infection based on a re-study of the Boeck-Bruusgaard material. Acta Derm Venereol (Stockh) 1955;35(suppl 34):3-368.

5 Clark EG, Danbolt N. The Oslo study of the natural course of untreated syphilis. J Chronic Dis 1955;2:311.

6 Grin EI. Epidemic syphilis in Bosnia: clinical and epidemiological observations on successful mass-treatment campaign. Bull WHO 1952;7:1-74.

7 Magnuson HJ, Thomas EW, Olansky S, Kaplan BI, DeMello L, Culter JC. Inoculation syphilis in human volunteers. Medicine (Baltimore) 1956;35:33-82. 